Décadrages Décadrages

cinéma, à travers champs Cinéma, à travers champs

$14 \mid 2009$

Cinéma et migration

\title{
La Suisse comme image, la Suisse comme son : Autour de Cyrill Schläpfer
}

\section{Alain Freudiger}

\section{(2) OpenEdition}

1 Journals

\section{Édition électronique}

URL : https://journals.openedition.org/decadrages/337

DOI : $10.4000 /$ decadrages.337

ISSN : 2297-5977

Éditeur

Association Décadrages

\section{Édition imprimée}

Date de publication : 1 avril 2009

Pagination : 91-104

ISBN : 978-2-9700582-9-8

ISSN : 2235-7823

\section{Référence électronique}

Alain Freudiger, «La Suisse comme image, la Suisse comme son : Autour de Cyrill Schläpfer », Décadrages [En ligne], 14 | 2009, mis en ligne le 10 avril 2010, consulté le 03 avril 2022. URL : http:// journals.openedition.org/decadrages/337 ; DOI : https://doi.org/10.4000/decadrages.337 


\section{La Suisse comme image, la Suisse comme son:}

\section{autour de Cyrill Schläpfer}

\section{par Alain Freudiger}

A l'occasion de la sortie de Die Waldstätte (2008), coffret de 4 CDs et 3 DVDs signé Cyrill Schläpfer, il nous a paru intéressant de questionner les liens qu'entretient la Suisse (comme représentation) avec le son, et d'aborder quelques aspects de la relation entre image et son lorsqu'elle est attachée à un Lieu.

Depuis sa "découverte» par l'Europe des Lumières et la construction des motifs qui la caractérisent, la Suisse est avant tout un paysage ou un lieu pittoresque, elle est liée prioritairement au visuel. Dès la fin du XVIII ${ }^{e}$ siècle en effet, les récits imagés de voyageurs côtoient les croquis, les gravures, les toiles, et partout, on fait de la Suisse une "peinture» de plus en plus typée. Au XIX ${ }^{\mathrm{e}}$ siècle, la Suisse des montagnes et des lacs est perçue comme miroir de l'âme, puis se contemple en panorama, et au tournant de la Belle Epoque, s'exporte en Suisse de carte postale. Elle commence à jouer de ses clichés à partir du milieu du $\mathrm{XX}^{\mathrm{e}}$ siècle, et finalement se "communique» en image redorée après la crise des fonds en déshérence... Mais à chaque fois elle se constitue d'abord comme une image.

Une image dont le son manque.

Certes, la Suisse abrite aussi un "peuple chantant», on n'y compte plus les chorales et les fanfares, ni le Ranz des vaches, jodel ou autre cor des Alpes qui lui sont traditionnellement associés. Mais au-delà de la musique, le bruit du pays lui-même demeure absent, sous-représenté. Et seul le vacarme créé par les fleuves, chutes d'eau ou avalanches est mentionné par les voyageurs $\mathbf{1}$. Paisible, romantique ou touristique, la Suisse semble ne pas faire de bruit, elle est muette (cinématographiquement) - c'est à peine si l'on entend le bruit du projecteur dans le fonctionnement du «spectacle»:

"La Suisse, à l'heure qu'il est [...] n'est plus qu'un vaste Kursaal, ouvert de juin en septembre, un casino panoramique, où l'on vient
1 Notamment les Chutes du Rhin, que Victor Hugo juge d'un "effroyable tumulte", et Lamartine "de l'orage imitant le fracas". A ce sujet, voir Reichler, Claude et Ruffieux, Roland (dir.), Le voyage en Suisse. Anthologie des voyageurs français et européens de la Renaissance au $X X^{e}$ siècle, Paris, Robert Laffont, Bouquins, 1998. 
2 Alphonse Daudet, Tartarin sur les Alpes, Flammarion, Paris, 1923, p. 117.

3 Concert-performance de Fritz Hauser aux thermes de Vals (2000), propositions sonores autour de Bernd Schurer dans le chantier du Lötschberg (2004), installations sonores dans le barrage pour le cinquantenaire de la Grande Dixence (2000), jusqu'au "Jardin de la violence " de l'Exposition nationale de 2002. La Société Suisse de Radiodiffusion produisit même, voici quelques années, un disque intitulé "Sounds of Switzerland". Sounds of Switzerland. Swiss Sound Mix. An Acoustic Tour of Switzerland. Produit par SRI - SRG SSR idée suisse, 2000.

4 "Ich bin hier in Luzern aufgewachsen. Das bedeutet, dass Schiffshörner zur Klangkulisse meiner Jugend gehörten. Ich mochte und mag diese Töne genauso wie Kuh- und Kirchenglocken oder Vogelgezwitscher. Für mich sind das vertraute Töne, mit denen ich Heimat definiere. Klänge sind wie Düfte und Farben oder die Silhouette eines Gebirgspanoramas: Das alles hat ganz bestimmte Wiedererkennungseffekte für alle. Und das macht Heimat aus. "Christian Hug, "Interview mit Cyrill Schläpfer über die Waldstätte", Luzerner Woche, 29.01.08 (notre traduction).

5 Ebranlement de la nation par la forte approbation en votation d'une initiative populaire visant à abolir l'armée suisse (1989), scandale de l'affaire des fiches (1989-1990), critiques virulentes sur le vide du $700^{\mathrm{e}}$ anniversaire de la Confédération (1991), choc lié au pavillon "la Suisse n'existe pas" à l'Exposition Universelle de Séville (1992)... se distraire des quatre parties du monde [...]. Avancez un peu dans le pays, vous ne trouverez pas un coin qui ne soit truqué, machiné comme les dessous de l'Opéra; des cascades éclairées à giorno, des tourniquets à l'entrée des glaciers et, pour les ascensions, des tas de chemins de fer hydrauliques ou funiculaires. $\mathbf{2}$

Et au $\mathrm{XX}^{\mathrm{e}}$ siècle, la Suisse peaufine son image discrète, silencieuse, ouatée. Pourtant la Suisse doit forcément exister comme matière sonore spécifique, et pas uniquement dans le registre musical, comme en témoigne depuis quelques années d'ailleurs la multiplication d'expérimentations sonores sur des lieux constitutifs de son identité3. Quel peut être le son de la Suisse, ou si l'on préfère, le son de l'image de la Suisse? Cyrill Schläpfer a tenté d'apporter quelques réponses à cette question.

"J'ai grandi à Lucerne. Ce qui signifie que les sirènes des bateaux appartiennent aux <coulisses> sonores de mon enfance. J'aime ces bruits exactement comme j'aime les cloches de vaches ou d'église, ou les chants d'oiseaux. Ce sont les bruits avec lesquels je définirais le pays natal. Les bruits sont comme les parfums, les couleurs ou la silhouette d'un panorama de montagne: ça a vraiment un effet de remémoration ou de re-connaissance pour tout le monde. Et cela constitue le pays natal.» $\mathbf{4}$

Il aborde en effet, dans son travail de cinéaste, à la fois la question de la "patrie» et celle du lien d'un son avec le paysage et le visuel. Ainsi, dans son précédent film, Ur-Musig (1993), Cyrill Schläpfer s'attache - 15 ans avant Heimatklänge (Stefan Schwietert, 2007) et en pleine époque de crise autour de la suissitude $\mathbf{5}$ - à montrer la musique traditionnelle suisse d'une manière nouvelle, à lui redonner en quelque sorte une dignité. Dans Die Waldstätte, il fait une sorte de "tentative d'épuisement» d'un lieu, en l'occurrence celui du Lac des Quatre-Cantons et de ses bateaux à vapeur, par le découpage et la (re)composition de ses sons. A travers ces «films» - constitués exclusivement d'images fixes, mais animés par une sorte de "mouvement de caméra» sur les photos - il pose certaines questions: Comment lier un son à un lieu? Comment filmer le son? Comment établir un rapport différent, nouveau, entre son et image? La problématique n'est évidemment pas spécifiquement suisse mais elle se décline de manière particulière ici - un pays qui, tout de même, a inventé pour ses trains les wagons-silence, un pays où des citadins établis à la campagne se plaignent des cloches de vaches ou d'église, un pays où il est interdit le dimanche de passer la tondeuse à gazon ou de jeter du verre vide... Un pays sans bruit et sans odeur? 


\section{Hiérarchie des sons, rapport à l'image}

Dans la hiérarchie implicite des sons, le bruit vient en général tout en bas, tant il apparaît comme non travaillé et même non humain; la parole, humaine par essence, vient au-dessus, et la musique, "quasi-divine», est au sommet. Dans cette même logique, l'image prime sur le son dans le courant traditionnel de l'histoire du cinéma.

Or dans ses productions, Cyrill Schläpfer émet des propositions qui provoquent des brouillages hiérarchiques : le bruit passe avant la musique, le bruit constitue de la musique, ou le bruit est mêlé à la musique. Les images, alors, sont soumises au bruit (Die Waldstätte) ou accompagnent le son (Ur-Musig). Son et image sont d'ailleurs dé-hiérarchisés jusque dans le produit: Ur-Musig est complété par un double CD qui n'est pas tout à fait la musique du film - Schläpfer le sous-titre "Original Tonspur des Films", c'est-à-dire "trace sonore» - et Die Waldstätte est un objet complexe, hybride, à la fois $\mathrm{CD}$ et DVD6. Avec cette relative porosité des supports d'expression et des catégories du son, on est à une limite en termes esthétiques, qui d'ailleurs rejoint toute une tradition "spiritualiste» de la Suisse, de Rousseau au Cantique Suisse (une Suisse qui ferait corps à la fois avec le paysage et avec le divin), et qui se rapproche de l'«œuvre d'art totale». Cependant, même si le travail de Schläpfer y tend, il n'y a pas tout à fait fusion, et certaines failles empêchent la formation d'une totalité indifférenciée. Sons et images sont liés mais jusqu'à un certain point seulement. Ainsi, on a du visible inaudible - la fumée du tabac des musiciens de Ur-Musig, par exemple - et de l'audible invisible - le vent, qui souffle fréquemment dans Ur-Musig.

Et si la volonté d'esthétisation est forte, jusqu'à "lisser» parfois des oppositions (entre la surface et la profondeur par exemple), il y a aussi des brèches, des fragilités, que ce soient les premières images de pièces sales, rouillées et usées du "DS Stadt Luzern» dans la série de portraits de vapeurs dans Akustische Portrait der Flotte, ou l'interlude constitué par le son suraigu du "MD Grütli» qui tranche avec la majesté des vieux vapeurs. Ou encore le musicien qui interrompt subitement son chant, comme mécontent de lui ou de la présence de la caméra, dans Ur-Musig. $\mathrm{Au}$ fond, ces films posent, outre la question des hiérarchies, la question des cloisons - et trouvent, dans ce questionnement même, quelques correspondances avec ce qui constitue la Suisse.

\section{En montagne: Ur-Musig}

Dans Ur-Musig, le parti pris esthétique est de ne pas séparer les sons purement musicaux des sons de l'environnement. Il en résulte un étonnant mélange de musique, de voix et de bruit, d'une harmonie et d'une
6 Le film principal, intitulé Waldstätte, a été présenté aux Journées Cinématographiques de Soleure en 2008. Quant au coffret, il propose 3 CDs auxquels correspondent 3 DVDs (Waldstätte. Dampfschiffsymphonie; Vierwaldstättersee. An Bord, Am Ufer; 5 Dampfschiffe. Akustische Portrait der Flotte), et un CD isolé (Das Lexicon). 
7 «Ich betrachte das natürliche Rauschen in einem schönen Blätterwald nicht als Musik (übrigens kein Musiker wäre imstande, je so ein schönes Werk zu komponieren). Aber angenommen, ein Mensch würde über sehr viele Jahre hinweg, an einem bestimmten Ort, speziell ausgewählte Baumsorten mit ganz unterschiedlichen Laubqualitäten anpflanzen; und macht dann viele viele Jahre später von seinem rauschenden Baumgarten eine Tonaufnahme, vielleicht würde er dies sogar bei spezifischen Windverhältnissen und Jahreszeiten tätigen: dann bezeichne ich dies als Musik. Dies ist in der Tat die entscheidende Frage und ist der wichtigste Prozess meiner Arbeit: wann wird aus Geräuschen Musik?». Frank von Niederhäusern, "Interview mit Cyrill Schläpfer ", RadioMagazin, juin 2008. force assez conséquentes. La cloche de vache, les bruits de pas ou le souffle du vent dans les bois du Muotatal ne sont pas plus déplacés que les notes de l'accordéon schwytzois ou les vocalises du jodleur. Avec ce film, Cyrill Schläpfer affirme que la Suisse a un son, que sa montagne a un son, et que ce son n'a pas besoin d'être décomposé, mais qu'il est mixte, que les frontières entre le bruit et la musique ne sont pas clairement définies. Le bruit n'est pas étranger à la musique - il la précède et il y participe, il s'y mêle ou il en constitue même 7 . Par ailleurs, les paroles s'intègrent au bruit et en deviennent: les cris des bergers, leurs appels, ont le même statut qu'un son de cloche, qu'un bruit de pas ou qu'une note d'accordéon. Et c'est ainsi que, dans le film, tout finit par faire musique. On se rapproche alors des concepts d'un John Cage, où le concert est donné avec les portes ouvertes aux sons de l'extérieur. Cette remise en cause des "frontières» est d'ailleurs aussi un questionnement qu'on peut mettre en lien avec la Suisse elle-même, pays institué par la frontière s'il en est. Pour autant, ce film ne représente pas une rupture par rapport à l'image traditionnelle de la Suisse : là où le son ouvre vers un ailleurs de l'image (y compris de l'image de la musique suisse), là où il se donne à écouter autrement, on retrouve cette volonté d'harmonie, d'union entre l'homme et la terre, et le «rousseauisme» passe par l'oreille - sans être d'ailleurs chassé par le visuel très travaillé et les plans très esthétisés du film (fig. 1-3).

Néanmoins, dans Ur-Musig, le son est là d'abord - et même là de toute éternité, ce que souligne le mot $U r$ : dans ce contexte, l'image, mal-

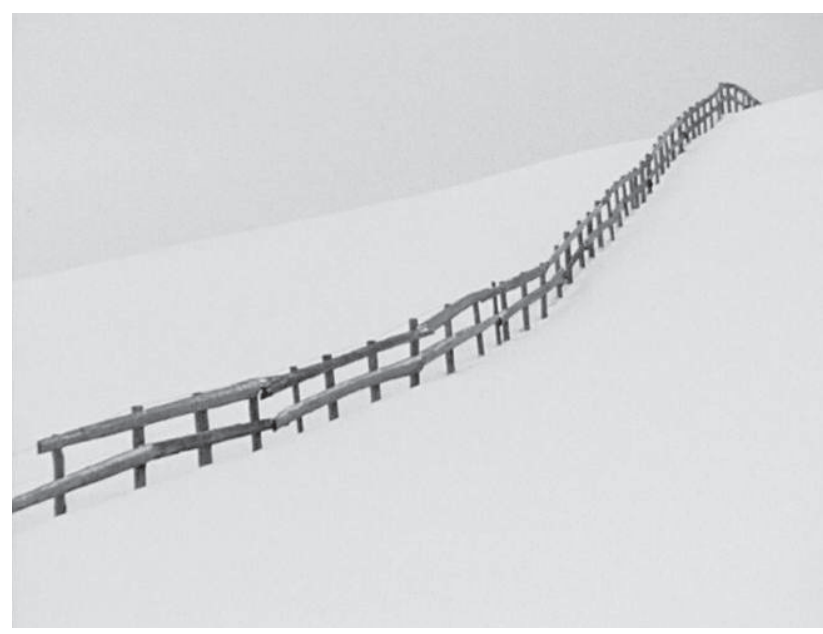

1

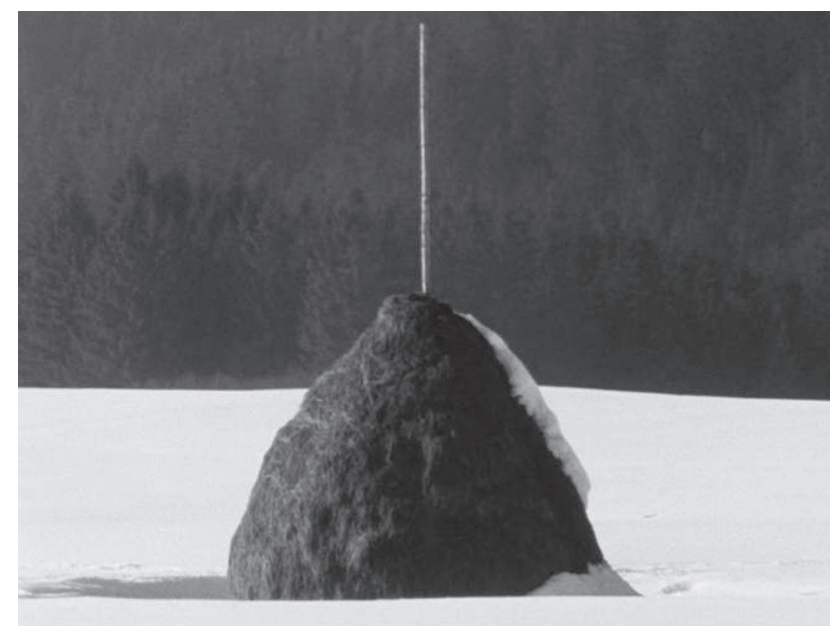

2 
gré sa beauté formelle, ne peut que constituer un accompagnement ${ }^{8}$. Quant à la musique à proprement parler, elle est parfois engloutie : ainsi, à la fin du premier CD Ur-Musig, le chant et la musique se terminent, remplacés par l'orage, la pluie, les cloches, les bêlements, les cris des bergers. Le son du paysage a absorbé l'autre son, mais dans une fluidité non conflictuelle, harmonieuse $\mathbf{9}$ - ce qui n'est pas loin de conférer au bruit la dignité de musique. Il faut noter d'ailleurs que le label de Schläpfer, CSR Records, a ensuite publié une série de disques d'enregistrements de cloches de vaches $\mathbf{1 0}$.

Les rapports entre son et image sont ainsi renégociés : la musique est comme un fond sur lequel viennent s'inscrire à la fois des bruits et des images. Par exemple, dans un plan du début, à des chants viennent se mêler des bruits d'outils (scie, hache), des cris d'animaux (coqs, vaches), qui apparaissent ensuite à l'écran : mais le point d'enregistrement n'établit pas de hiérarchie sonore explicite, et l'image ne tranche pas non plus, un plan ne l'emportant pas sur l'autre. En outre, images et sons ne coïncident parfois pas, ou alors seulement partiellement: ainsi, sur un air d'accordéon, on entend une ou deux cloches de vaches, mais pas assez par rapport au troupeau qu'on voit à l'écran; on voit aussi des bouches qui articulent mais sans qu'on puisse entendre ce qu'elles disent. Le son et l'image se décalent, se disloquent ou s'absorbent. A la fin du film, alors que le chant des bergers, les cloches et les bêlements des moutons se mêlent en un tout sonore, un plan général de troupeau dans un pré finit par un fondu sur le pré seulement, comme si le troupeau et
8 La musique, d'ailleurs, est incarnée par des visages plutôt que par des gestes: le son et l'image ne sont pas dans un rapport technique de production, mais plutôt dans un rapport poétique d'émanation.

9 Une harmonie anhistorique qui fait écho au "Ur" primitif et à la mythologie rousseauiste.

10 Montées à l'alpage, troupeaux au pâturage, désalpes, etc. S'Glüüt, vol. 1 à 9 . Collection "True Tone", CSR Records, 1993-2006.

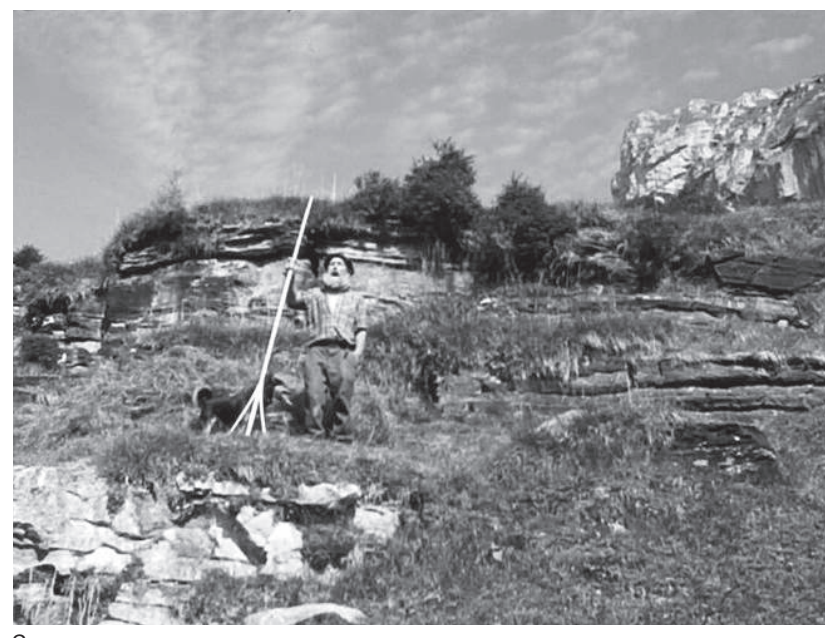




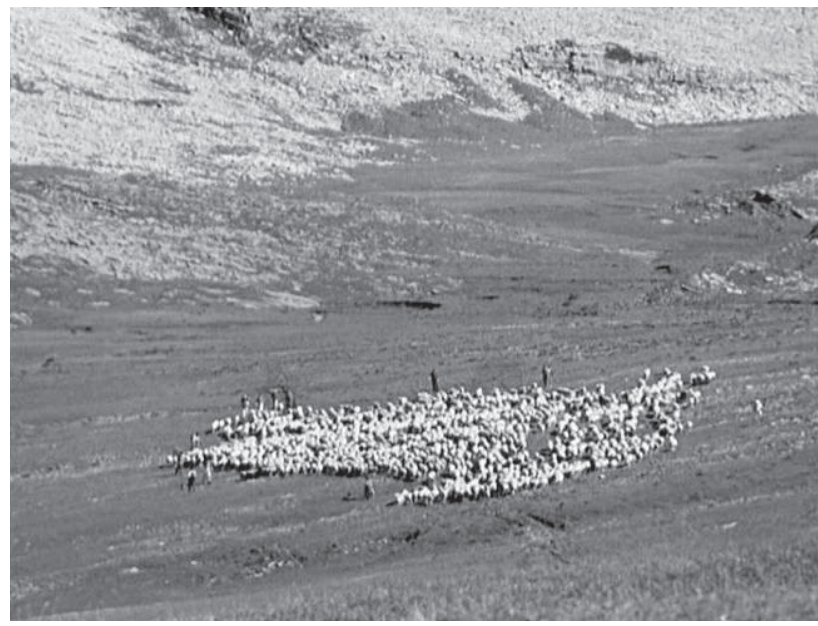

4

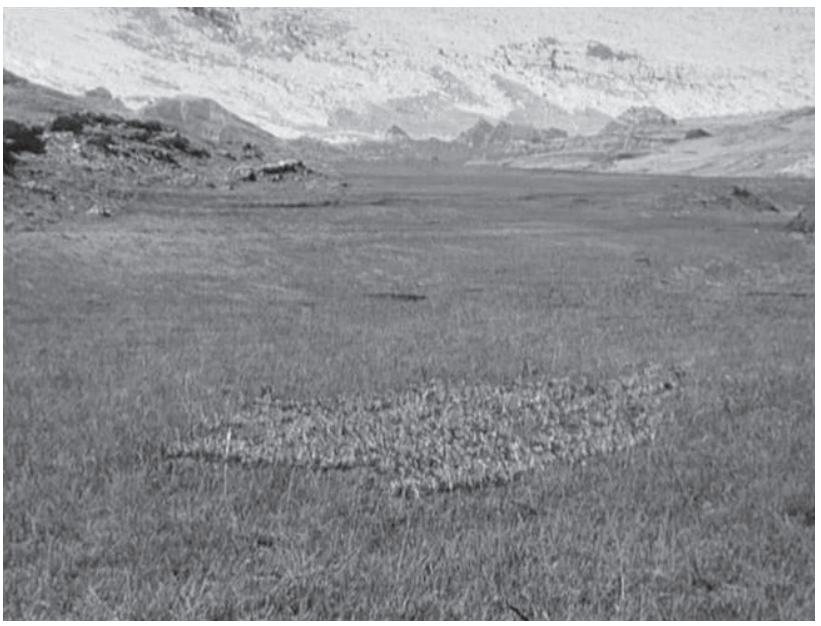

5

les bergers se fondaient fantomatiquement dans le paysage (fig. 4-6). Et finalement, pendant le générique de fin la seule "musique» consiste en cloches de vaches, toujours plus fortes et intenses.

\section{Sur l'eau: Die Waldstätte}

Avec Die Waldstätte (2008), Schläpfer pousse son travail encore plus loin puisque, comme nous allons le voir, il place le bruit au centre, réduit l'image à une expression minimum et supprime la musique en tant que telle, pour la faire surgir du bruit même. Dans cette «réorganisation», le

11 "These are among the familiar sounds [of my environment] for me like my mother tongue, church bells, cowbells, insects, birds, etc... It's horrible to even think of not being able to hear these sounds anymore or being concealed by the generic noise of our pornographic civilization. That's why I recorded those sounds without the commuters and tourists, hikers, skiiers and nature lovers, because they mask or even eliminate its magic." Bart Plantega , " Jesus! By what devil I have been ridden...?? ?. Interview with Cyrill Schläpfer", Radio Patapoe FM, Amsterdam, mars 2008. www.csr-records.ch/ presse-archiv-i.html.

12 " Nicht die Bilder sind wichtig, die Komposition zählt., Cyrill Schläpfer äusserte diese Worte vor der Projektion seiner Dampfschiffsinfonie Die Waldstätte an den diesjährigen Solothurner Filmtagen. "Benedikt Sartorius, "Der Zweifler und die Dampfschiffe", Der Bund, 19.02.08. supposément inerte s'anime, tandis que l'image en mouvement se fige et que l'homme s'efface du champ.

L'ensemble est composé essentiellement de bruits de bateaux à vapeur - dont il faut relever l'exceptionnelle qualité sonore, les sons tendant vers l'byperréel - qui se déclinent en un large spectre et une riche variété de sons (pompes, sirènes, eau, machines, etc.). Ces sons d'ailleurs, sans être "purs", sont non-parasités, les parasites étant triés: cloches, pluie, oiseaux sont intégrés, tandis que d'autres (avions, touristes, etc.), indésirables, sont écartés, coupés au montage11. Les sons sont alors réarticulés sous forme de: narration (Vierwaldstättersee. An Bord, Am Ufer), portraits (5 Dampfschiffe. Akustische Portrait der Flotte), symphonie (Waldstätte. Dampfschiffsymphonie) et inventaire (Das Lexicon). Ils dépassent donc leur statut de bruit pour devenir une sorte de langage.

C'est que le son prime sur l'image12. Les films DVD de Die Waldstätte sont un montage d'images fixes, mais avec une sorte de "mouvement de caméra» sur les photos dans différentes directions, avec des 


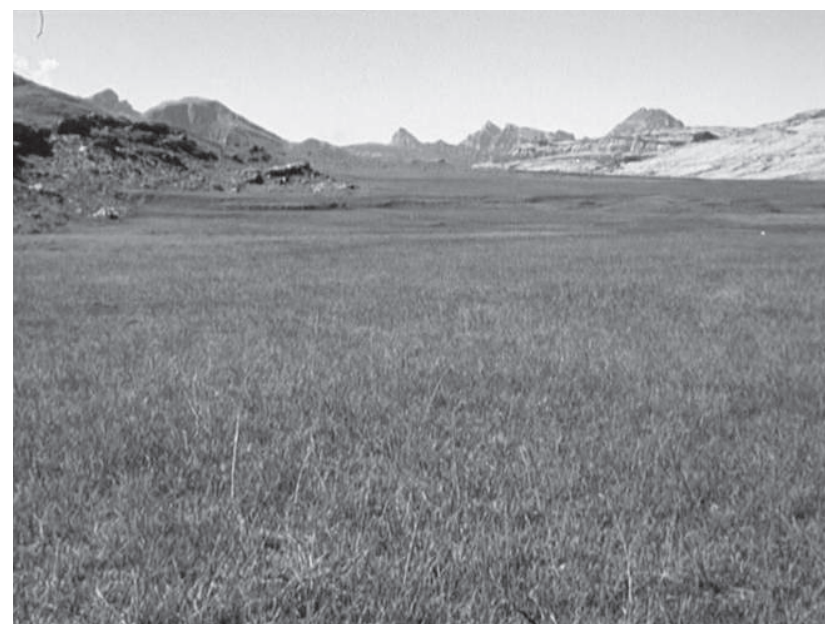

6

accélérations et des ralentissements, et des zooms avant ou arrière. Il n'y a jamais véritablement de plan fixe: on "navigue» dans des images manipulées par ordinateur avec des effets de déviation d'angles et des trajectoires calculées typiques d'une esthétique "computériste». Cette esthétique imprime un rythme et une direction en forme de dérive - on bouge dans les plans un peu comme on pourrait se déplacer avec un bateau. Le travail sur les couleurs est marqué, tout comme celui sur les cadrages: formellement, il y a une forte volonté d'esthétisation (fig. 7-9). Sauf moment particulier, la durée des plans est grosso modo toujours la même, soit 9 ou 10 secondes: cette régularité permet une plus grande attention aux «irrégularités» de la construction sonore. Par moments, des effets de brillance sur l'eau ou dans le ciel donnent l'illusion d'un mouvement interne à l'image. Or, le kitsch de cet effet contrastant avec la finesse du traitement acoustique, il est étonnant que Schläpfer accepte pour le visuel ce qu'il refuse pour le son:

«Pour la symphonie, j’ai utilisé exclusivement les sons des bateaux (sifflets, sirènes, métal, machines...), des bruits d'eau et des ambiances naturelles (oiseaux, vent, tonnerre, pluie, etc.). J'ai seulement fixé certaines tonalités plus bas, pour essayer d'harmoniser ou de trouver une harmonie en corrélation avec ce qui précédait ou suivait. A part ça, j'ai fait beaucoup de coupes et de montage (18000 coupes pour la symphonie) et j'ai inversé certains sons. Mais il n'y a absolument aucun ajout de sons électroniques, ni d'effets comme de la réverbération, des filtres ou n'importe lequel de ces gadgets de producteur de son.»13
13 "For the symphony, I used exclusively sounds from the ships (whistles, horns, metal, machines...) and water and natural ambience (birds, winds, thunder, rain, etc.). I only pitched the sounds lower, trying to harmonize or find the harmony in correlation with what was going on before or after. Besides that, I did a lot of cutting and editing, (18,000 cuts in the symphony) and I reversed some of the sound. But there is absolutely no addition of electronic synthesizer sound, or effects like reverb, filtering or any of those sound-producer gadgets or enhancers. ", Bart Plantega, op. cit. (notre traduction). 


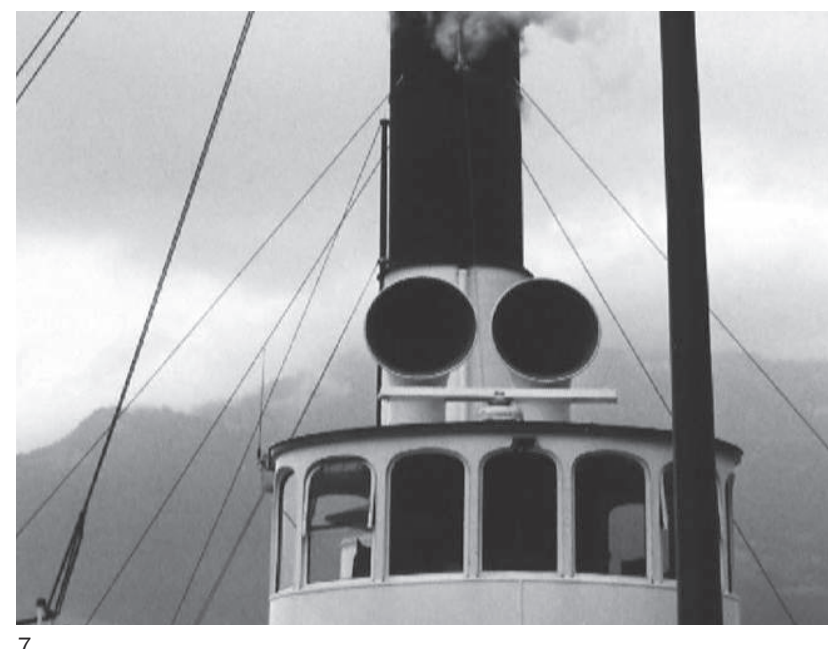

On peut supposer que la matière visuelle est considérée comme moins noble, puisque contrairement à la matière sonore elle n'a pas besoin de rester intacte, ce qui illustre encore une fois la primauté du son. Quant au lien entre image et son, la coïncidence rythmique est parfois frappante, notamment avec les coups de sirène ou les cloches qui ponctuent des changements de plan - et on est souvent dans le registre illustratif, même si ce n'est pas systématique: à un bruit métallique répond un plan sur une passerelle, à des bruits d'eau et de roue à aubes des plans d'eau et de roue à aubes... Si c'est une "faiblesse» cinématographique, cela asseoit définitivement la prééminence du son - ce que souhaite Schläpfer qui ne s'en cache pas.

C'est que le détail est dans le son, non dans l'image - la précision aussi, et l'événement de même. Dans Vierwaldstättersee, c'est l'absence de vagues et de bruit de bateau qui fait percevoir concrètement le calme du plan sur le ponton - et c'est par le son qu'arrive d'abord l'orage. Cependant, des correspondances entre son et image peuvent jouer à un autre niveau. Au détail peut répondre un autre détail, une autre précision : un son clair, précis, fait par exemple écho à des plans de détails techniques. Par contre, au vu du parti pris visuel, il se produit tout de même une sorte de décalage entre le bruit, perçu comme vivant, et l'image fixe, qui relève plutôt du non-vivant. C'est dans cet interstice que viendra se loger quelque chose qui relève du fantomatique, nous y reviendrons. Mais on peut noter déjà que sur le plan sonore, l'humain manque, et que sur le plan visuel, il est rare également (mis à part dans le DVD 5 Dampfschiffe où l'équipage est assez présent visuellement). Parfois seulement 


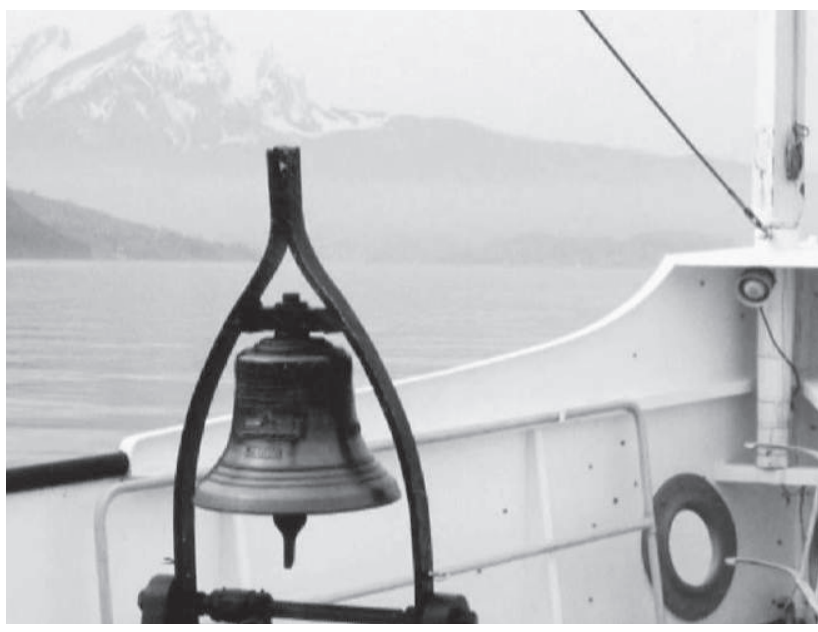

8

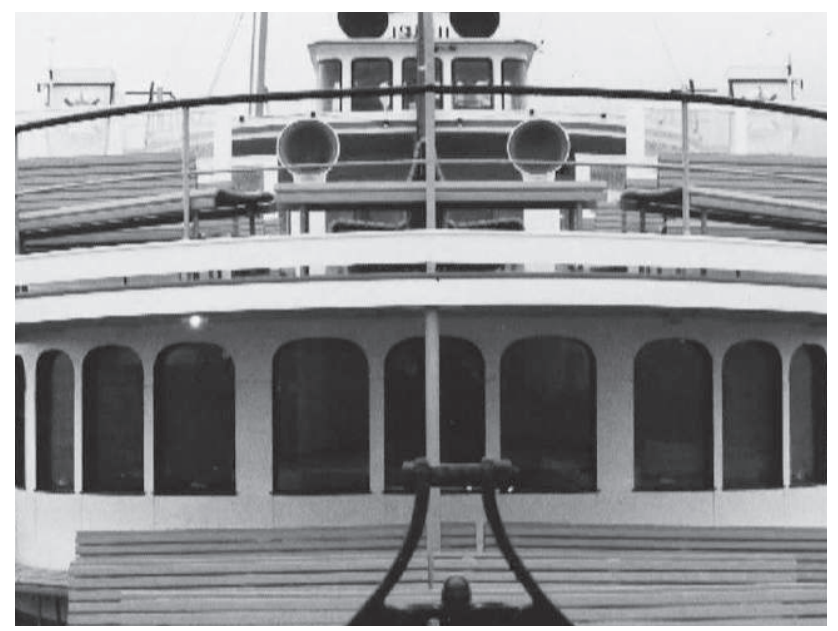

9

on aperçoit des passagers, mais ils sont toujours en grappe, jamais individualisés, et pris dans le décor, presque de trop. L'homme est absent de Die Waldstätte: ce sont les éléments, les machines et les animaux qui figurent.

\section{Une narration: Vierwaldstättersee. An Bord, Am Ufer}

Vierwaldstättersee est un voyage acoustique sur le Lac des QuatreCantons, qui oscille entre les rives et l'eau, les quais et les bateaux. Par le jeu du contraste entre sons graves et aigus, entre souffle et grondement, entre proche et lointain, les sons se chassent les uns les autres, se mêlent ou se couvrent. Mais dans cette masse sonore principale demeure toujours un peu d'eau, parfois très légèrement, comme une trace persistante. Faisant office de liant, de corps, l'eau, d'abord clapotis périphérique et discret, peut tout à coup s'imposer à nouveau lorsque les roues à aubes d'un bateau approchent, avec des bruits immenses et puissants qui submergent les autres sons - puis disparaître à nouveau au second plan lorsqu'un bateau s'éloigne. L'ensemble du disque/film constitue de fait une narration: le départ, l'orage, les haltes, la pause hivernale... Mais cette linéarité apparente laisse place à d'autres axes de lecture à travers la grande variation d'intensité des sons et de leur volume. Le bruit, ainsi, délimite l'espace et dessine les places respectives. Quant au mouvement sur des photos du lac, en forme de lente dérive, il est dynamique, avec des moments de calme et de redémarrage, jusqu'à l'arrivée de l'orage où les plans se font soudain très sombres, et où une brume rend la vision trouble: ce qui confirme que l'on est dans un rapport mimétique direct, 
14 Ce côté "abyssal" répond d'ailleurs sous forme sonore à l'image romantique de la Suisse et de ses gouffres.

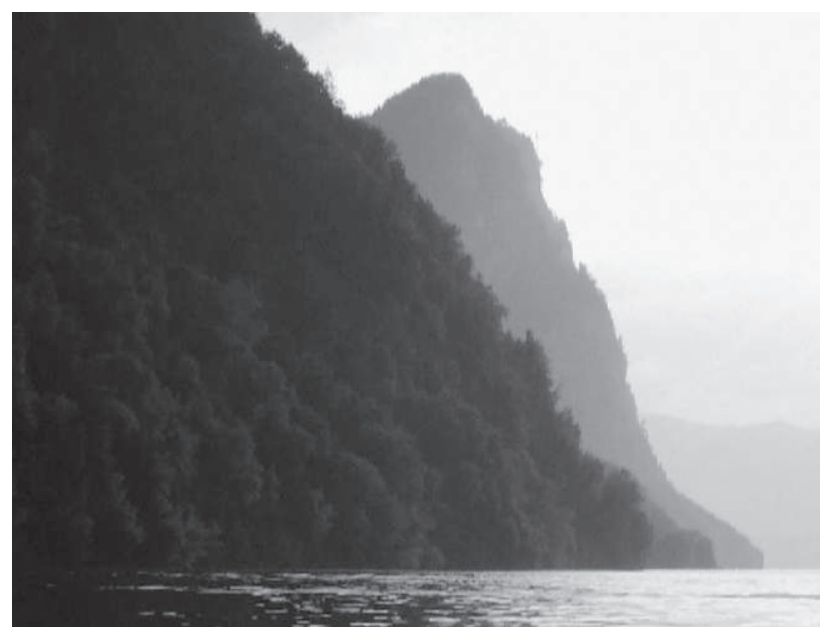

que l'illustration est complète. Mais ce n'est pas une illustration «typique»: ce lac, Schläpfer le rend plus sauvage et presque "archaïque», loin du tourisme et du folklore. Il l'écoute, l'observe et en propose une «restitution» (fig. 10-11).

\section{Une symphonie: Waldstätte. Dampfschiffsymphonie}

Avec Dampfschiffsymphonie, le bruit devient clairement musique, par le truchement de la composition d'une pièce de musique concrète. Dédiée à «Moby Dick», Dampfschiffsymphonie pousse plus loin le côté sauvage et archaïque et joue beaucoup sur les profondeurs, les bruits sous-marins inquiétants, avec en contrepoint des sifflets lointains et des bruits d'eau en surface ${ }^{\mathbf{1 4}}$. Les ruptures sont plus sèches: un chant de mouette est soudain suivi par des sons d'abysses; des cloches (aériennes) viennent ponctuer un "engloutissement». Jouant sur un axe de verticalité spatial (haut-bas) autant que sonore (aigu-grave), un monte-charge contraste avec un bruit d'ancre jetée à l'eau. Au niveau visuel, le film est aussi plus «travaillé» que les autres, plus irréel, avec notamment des blancheurs "spectrales»: plans récurrents avec un motif de bateau-fantôme (fig. 12), noir-blanc, images inversées en négatif... On retrouve donc le fantomatique. Mais le film est plus travaillé encore en ce qui concerne les correspondances que les images entretiennent avec la musique (correspondances plus rythmiques qu'illustratives). Quant aux bruits, ils se répondent et forment un ensemble quasi "symphonique» avec des équivalents de percussions (coups, choc), de cordes (craquements, grin-

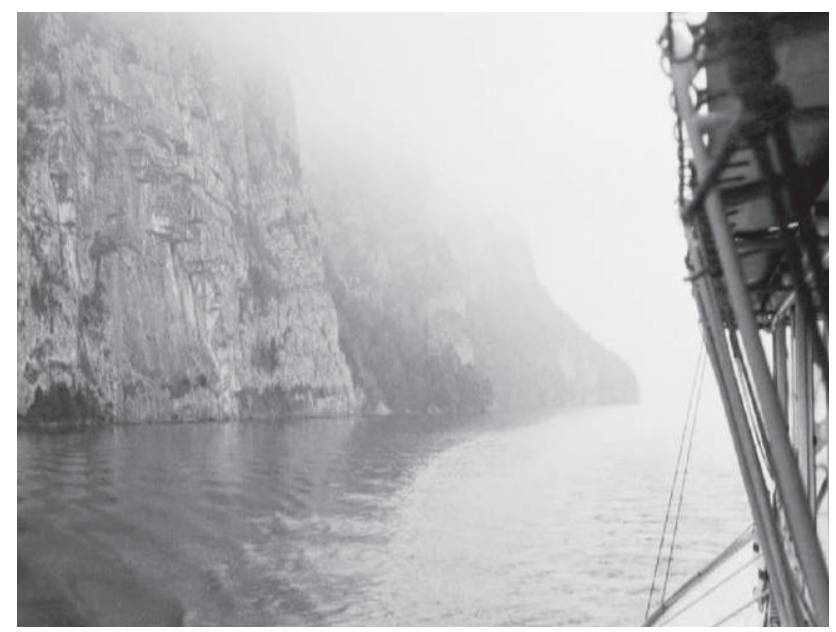

11 


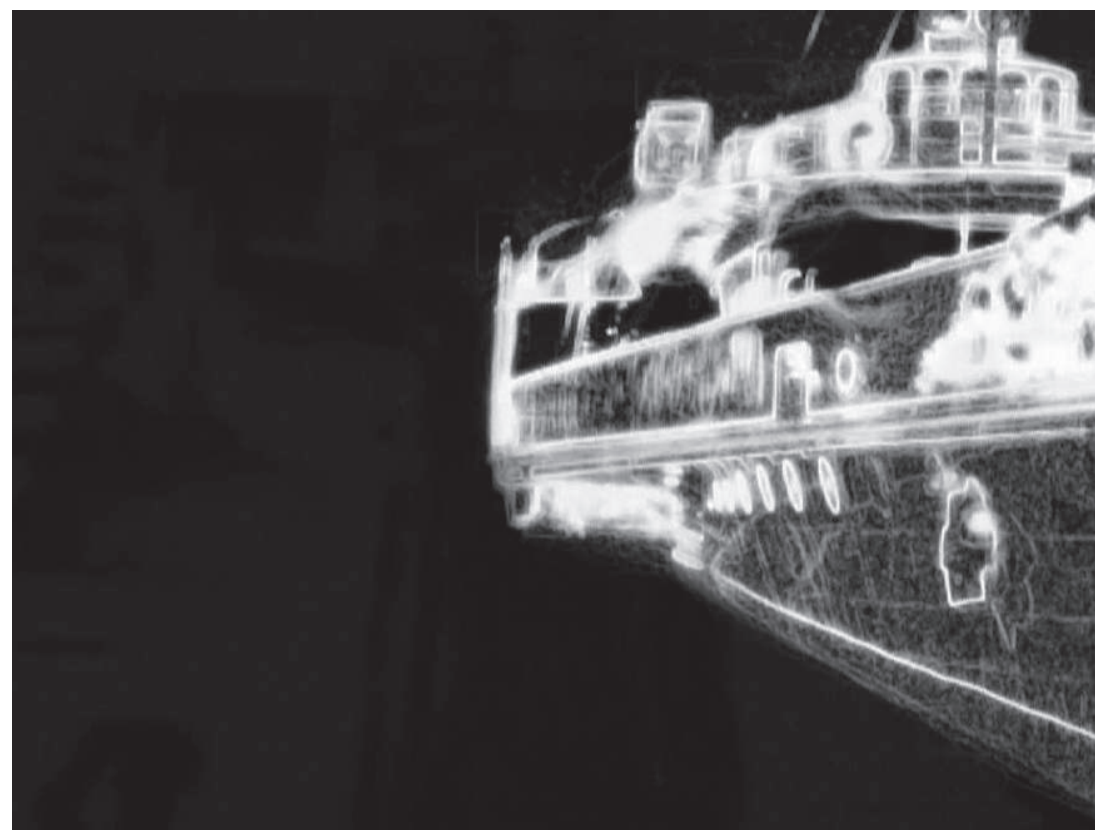

12

cements) et de vents (sirènes, jets de vapeur). Ils changent réellement de statut, pour devenir musique. Mais, de par le traitement qui est ici apposé aux sons, on est déjà dans un registre différent des autres enregistrements de Die Waldstätte, puisqu'il s'agit d'une composition musicale.

\section{Un inventaire: Das Lexicon}

A l'opposé, Das Lexikon est le disque le moins composé de la série ${ }^{\mathbf{1 5}}$. Mais il est travaillé d'une autre manière: il constitue un inventaire, les bruits ici se laissant détailler un à un, plage après plage. Ainsi, le son se laisse répertorier, domestiquer, documenter. On sort du registre du film, puisqu'il n'y a pas d'équivalent DVD. Mais le disque se présente comme un catalogue des pièces détachées qui auraient servi à la composition. Ici, tout est séparé, classé - c'est peut-être aussi une manière de toucher à la sonorité de la Suisse, ce pays "propre en ordre» - et c'est pratiquement le seul lieu où il y a de la place pour la voix humaine, avec des plages où on entend des annonces du capitaine: la voix se laisse répertorier et délimiter comme n'importe quel autre bruit de bateau, signe de plus, s'il en fallait, de son statut «déchu».
15 Si la symphonie de musique concrète relève clairement de la "composition", Cyrill Schläpfer considère que les autres disques en constituent également: "Still, I consider it a composition, since you will not find such pure and unadultered sound in nature. By which I mean: there is always acoustic pollution like planes, cars, tourists, cash-machines, farts, crunching french-fries, microwave ovens, cellular phones...." "Jesus! By what devil I have been ridden...??", interview avec Cyrill Schläpfer par Bart Plantega pour Radio Patapoe FM, Amsterdam, mars 2008. 


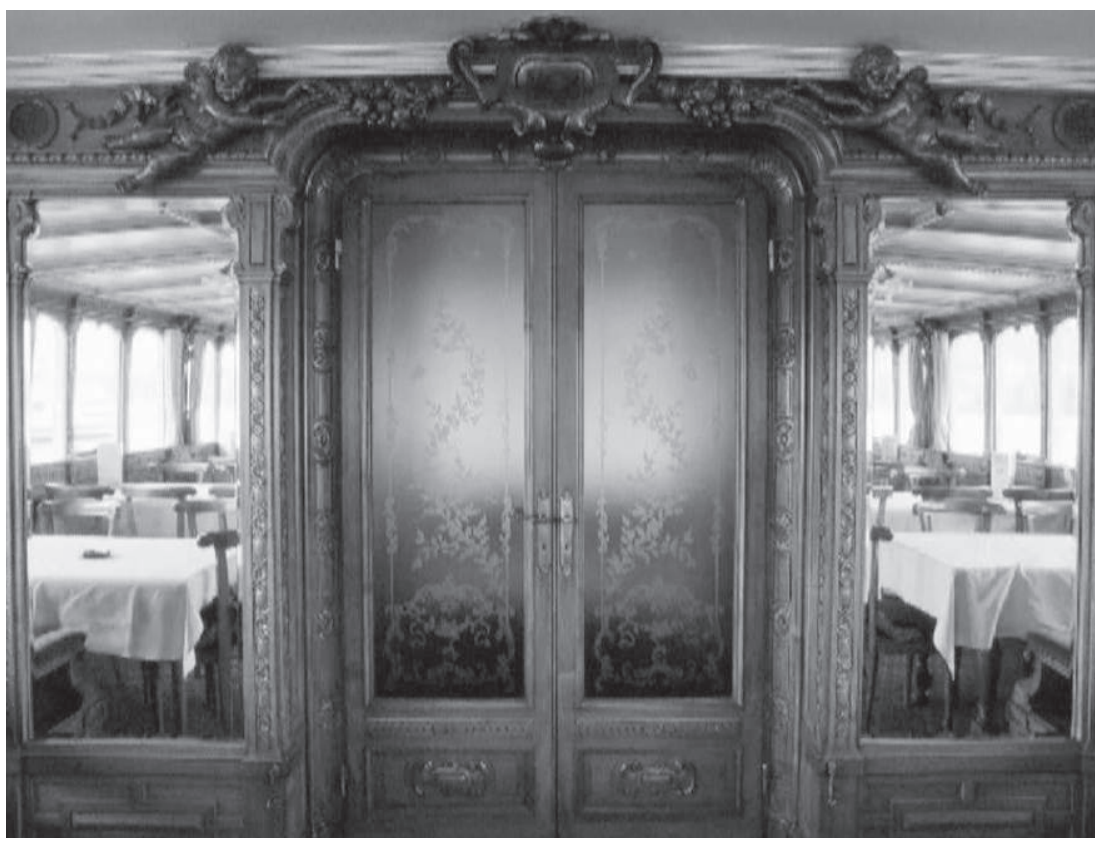

13

\section{Des portraits: 5 Dampfschiffe. Akustische Portrait der Flotte}

Enfin, dans 5 Dampfschiffe, chaque plage ou chapitre est consacré à un navire différent de la flottille. On part du bruit pour rejoindre le genre du portrait, par le biais d'une sorte de métonymie (tel bruit renvoie au bateau dans son entier). Mais les bruits finalement se combinent pour manifester une Présence: par les grondements et les claquements, le son devient presque animal - comme un monstre qui siffle, sonne et résonne. Les souffles profonds évoquent quelque difficulté respiratoire, tandis que les pompes et les pistons figurent des battements de cœur. On sent alors assez clairement la volonté de brouiller d'autres frontières, entre machine et animal par exemple. En outre, par les sirènes et leurs résonances, qui évoquent quelque prise de parole, Schläpfer confère aux bruits une dignité. La présence humaine est minime. Ce sont les bateaux qui sont les vrais "personnages». Et leur présence s'étend: par un jeu sur les textures, sur les angles ou sur les lignes, le film remet en cause les limites du bateau qui est alors, à l'instar du portrait 3 («DS Schiller»), tellement lié à l'environnement, au lac, aux oiseaux qu'il disparaît même parfois au niveau sonore, effacé par l'eau, n'existant plus qu'en creux - avant de se réveiller par un brusque son puis de réapparaître à l'image. Par moments, un montage rapide de photogra- 
phies rappelle quelque vieux dispositif optique ou le fonctionnement des diapositives : bruit régulier, ponctuations, fondus au noir... On a alors vraiment affaire à une Présentation - Présentation dont à nouveau l'homme ressort comme absent, à l'image de ces plans sur les magnifiques salons intérieurs vides (fig. 13) et d'où émane, une fois de plus, une impression de fantomatique.

\section{Le son: une construction}

Or le son, s'il témoigne d'un présent, est aussi comme une remontée du passé $(U r)$, qu'il rejoue. Est-ce pour cela qu'il est en lien avec le fantomatique? Le son reproduit est-il déjà une mort, une absence, comme le "ça a été» de la photographie selon Barthes? On connaît, en particulier, la puissance d'évocation du bruit. Son lien avec l'image passerait alors, peut-être, non par une relation directe, mais médiatisée par le fantasmatique. La Suisse du son, alors, ne serait pas plus «réelle» que celle de l'image.

Quoi qu'il en soit, même si la Suisse est devenue sonore grâce au travail de Cyrill Schläpfer, il s'agit d'un son "construit»: la montagne, le lac, débarrassés de leurs oripeaux «touristiques», et presque de leur présence humaine (mais c'est ce "presque» qui fait le fantomatique, et non l'absence complète). Or ce son, on ne sait même plus s'il faut l'appeler bruit, tant les hiérarchies sont brouillées. Le bruit n'est plus inférieur à la parole, puisqu'il peut communiquer (narrer) et symboliser (déterminer des places respectives). Il s'élève même jusqu'au niveau de la musique, que ce soit par mélange (dans Ur-Musig) ou par composition (la musique concrète de Dampfschiffsymphonie). La parole, elle, s'intègre comme bruit, mais n'a plus de prédisposition fondamentale à communiquer du sens (c'est un élément sonore parmi d'autres dans Das Lexicon). Quant à la musique, elle disparaît parfois, masquée par le bruit, ou survient à un niveau supérieur, celui de l'ensemble (Ur-Musig), comme une composition qui engloberait le tout. Au niveau de l'image, la Suisse est devenue tableau, panorama ou carte postale; il semble que la Suisse sonore se constitue en symphonie, film auditif ou installation sonore, c'est-à-dire en un son qui n'a rien non plus de "naturel»- mais avec pourtant une inventivité et une liberté qui n'est que rarement laissée à son image. 


\section{Die Waldstätte (2008)}

Coffret 4CD-3DVD. Contient:

Waldstätte. Dampfschiffsymphonie, CD-DVD, 70 min.; Vierwaldstättersee. An Bord, Am Ufer, CD-DVD, 79 min. ; 5 Dampfschiffe. Akustische Portrait der Flotte, CD-DVD, 74 min. ; Das Lexikon. 99 Töne, Klänge, Geräusche der DS URI, DS Unterwalden, DS Schiller, DS Gallia, DS Stadt Luzern, MS Rütli und MS Reuss, CD, $78 \mathrm{~min}$.

CD Audio Stereo, DVD 5.1 Surround Dolby Digital. Réalisation: Cyrill Schläpfer. Image: Cyrill Schläpfer. Son: Cyrill Schläpfer. Production: CSR Records. Collection Truetone, Vol. X-XIII. nº commercial: 90732

\section{Ur-Musig (1993)}

Réalisation: Cyrill Schläpfer. Production: Cyrill Schläpfer, CSR Records / Schwing Produktion. Image: Thomas Krempke. Son: Dieter Meyer, René Zingg, Cyrill Schläpfer. Montage : Jürg Hassler. Interprétation: Rees Gwerder, Jakob Alder, Jakob Düsel...

Disponible en DVD, CSR 91511, ou en deux versions CD, CSR 2xCD91512, sur www.csr-records.ch 